\title{
ON MELTING OF TWO-DIMENSIONAL MONOLAYER FILMS
}

\author{
A. Patrykiejew, T. Zientarski \\ Department of Chemical Physics, Faculty of Chemistry, MCS University \\ Pl. M. Curie-Sklodowskiej 3, 20-031 Lublin, Poland
}

AND K. BINDER

Institut für Physik, Johannes Gutenberg Universität Mainz

55099 Mainz, Germany

(Received March 28, 1996)

The melting of two-dimensional films formed on the (100) fcc crystal is studied by Monte Carlo simulation. The results obtained suggest that in systems with only weakly corrugated surface potential, exhibiting the hexagonal close packed solid structure, the melting transition is followed by the Ising-type transition as predicted by the theory of Nelson and Halperin. In the case of highly corrugated surface potential, the film forms registered structure which disorders gradually as the temperature is raised.

PACS numbers: 82.65. $-\mathrm{i}, 64.60 .-\mathrm{i}$

The mechanism of melting and disordering of two-dimensional adsorbed solid phases is a subject of considerable current interest and hot controversy [1-3]. One of the questions is the adequacy of the picture provided by the theory developed by Kosterlitz, Thouless, Halperin, Nelson and Young (KTHNY) [4-7] to real experimental systems. The hitherto collected experimental data $[1,8-10]$ as well as the results of numerous computer simulations $[1,11-14]$ seldom lead to unambiguous conclusions. The KTHNY theory predicts that the melting of two-dimensional solid phase on a "flat" (noncorrugated) surface is a two-stage continuous process. In the first stage, due to dissociation of dislocation pairs, the system looses the quasi-long-range positional order, but retains the quasi-long-range orientational order. This corresponds to the transition from the solid to the hexatic phase. The second stage is connected with the disclination-unbinding transition, occurring at higher temperature and leading to the formation of isotropic liquid phase. This scenario is considerably altered by the presence of periodic substrate potential and the actual mechanism of melting depends on the symmetry and the size of the substrate surface lattice as well as on the amplitude of periodic variations of the gas-solid potential. In general, the presence of periodic potential is expected to wash out the disclination-unbinding transition, and hence the melting becomes 
a one-stage process. This transition may be continuous, as for the flat surface, or first-order. The only exception is the melting of a hexagonal adsorbate film on a square lattice characterized by weak corrugation of the gas-solid potential. In this case the KTHNY theory predicts that the disclination-unbinding transition is replaced by an Ising-like transition [6]. Thus, one expects that there are two "liquid" phases with different orientational symmetry.

In this paper we report the results of Monte Carlo simulation for a series of two-dimensional adsorbed films on a square lattice, the (100) face of a simple fcc crystal, and exhibiting different corrugation of the gas-solid potential. The mutual interaction of adsorbed atoms is described by the $(12,6)$ Lennard-Jones potential, $u(r)$, truncated at a certain distance, $r_{\max }$. Throughout this paper we assume that the cut-off distance is set at $r_{\max }=2.5 \sigma$, as it was also assumed in our earlier paper dealing with three-dimensional systems [14]. The adsorbed atoms are also subject to the surface potential, $V_{2 \mathrm{D}}(\tau)$, being a function of the two-dimensional vector $\tau=(x, y)$, which specifies the position of an atom relative to the surface lattice unit cell. The surface potential is periodic and in the three-dimensional system can be calculated using the Fourier expansion developed by Steele $[15,16]$,

$$
V_{3 \mathrm{D}}(z, \tau)=\varepsilon_{g s}^{*}\left[V_{\mathrm{eD}}^{0}(z)+V_{\mathrm{b}} \sum_{q} V_{3 \mathrm{D}}^{q}(z) f_{q}(\tau)\right] .
$$

In the above equation the summation runs over all reciprocal lattice vectors $q$ and $V_{\mathrm{b}}$ is an adjustable parameter allowing to change the periodic part of the gas-solid potential. The Fourier coefficients $V_{3 \mathrm{D}}^{q}(z)$ and the functions $f_{q}(\tau)$ can be found in Ref. [15].

Now, we assume that in two-dimensional systems the gas-solid potential takes the following form:

$$
V_{2 \mathrm{D}}(\tau)=\min _{z}\left[V_{3 \mathrm{D}}(z, \tau)\right],
$$

which ensures that the corrugation effects are exactly the same as in the related [14] three-dimensional model, providing that all parameters are the same. One can expect, however, that for a given corrugation parameter, $V_{b}$; the two-dimensional system will exhibit slightly higher tendency towards localized adsorption than its three-dimensional counterpart. This is so, because by suppressing the out-of-plane motion we eliminate the possibility of a better accommodation of the hexagonal phase. In three dimensions some particles may stay at larger distance from the surface but the interparticle distances may still remain practically unchanged. On the average, the area occupied by a single particle in the three-dimensional system is expected to be slightly smaller than in the strictly two-dimensional system.

We consider a series of systems with the adsorbate atoms of the size, $\sigma^{*}=$ $\sigma / a=0.9$, and the density equal to the density of completely filled registered monolayer, $\rho^{*}=\rho \sigma^{* 2}=0.81$ and with different effects of the gas-solid potential corrugation (controlled by the value of the parameter $V_{\mathrm{b}}$ ). Then, we assume that $\varepsilon_{g s}^{*}=2.0$, in agreement with the assumption of Ref. [14]. One should note that unlike in studies of two-dimensional systems on noncorrugated surfaces, where the strength of the gas-solid potential is irrelevant, here it influences the height of potential barriers for surface diffusion. 
From the simple ground state calculations it follows [14] that for sufficiently low values of $V_{b}$, the systems considered here form the hexagonal close packed (hcp) solid phase at low temperatures. On the other hand, when $V_{\mathrm{b}}$ exceeds a certain limiting value, $V_{\mathrm{b}, \mathrm{lim}}\left(\sigma^{*}\right)$, the stable low temperature structure corresponds to the registered $1 \times 1$ phase. From the observations made for three-dimensional systems it is clear that for intermediate values of $V_{b}$ the uniaxially ordered phase is likely to appear. This possibility has not been taken into account in the ground state calculations and we cannot determine the limits of $V_{\mathrm{b}}$ embracing the region of stability for this phase. The predictions based on the simple geometrical argument as proposed by Bruch and Venables [17] may be helpful in determining whether such phase is likely to appear for a given $\sigma^{*}$.

Since our aim here is to study subtle changes in the system structure, we need to use appropriate tools. The usual calculation of such thermodynamic properties like the gas-gas and gas-solid contributions to the total system energy and the heat capacity are very useful in determining the appearance of different phase transitions, but do not give precise information concerning the microscopic changes in the system structure. Therefore, apart from the quantities mentioned above we also record the radial distribution function, $g(r)$, and the bond-orientational order parameters, $\psi_{k}$ and $\psi_{k}^{\mathrm{e}}$, defined by

$$
\psi_{k}=\left|\frac{1}{N_{\mathrm{b}}} \sum_{m} \sum_{n} \exp \left[\mathrm{i} k \phi_{m n}\right]\right|, \quad k=4,6
$$

and

$$
\psi_{k}^{\mathrm{e}}=\left|\frac{1}{N_{\mathrm{b}}} \sum_{m} \sum_{n} \cos \left[k \phi_{m n}\right]\right|, \quad k=4,6,
$$

where the first sum runs over all particles in the system, the second sum over all nearest neighbors of the particle $m, \phi_{m n}$ is the angle between the bond joining particles $m$ and $n$ and an arbitrary reference axis, chosen here to be the $x$-axis of the surface lattice, and $N_{\mathrm{b}}$ is the number of bonds in the system. Basically, in an ideal situation, when only pure phases are present, we can readily determine their nature by looking at the behavior of the bond-orientational order parameters. Namely, in the disordered, liquid or gas phase, all these parameters should be equal to zero since all possible mutual orientations of bonds appear in the system with the same probability. In the registered phase of square symmetry, either $1 \times 1, \sqrt{2} \times \sqrt{2}$ etc., we expect $\psi_{4}=\psi_{4}^{\mathrm{e}} \approx 1$ at sufficiently low temperatures, and both $\psi_{6}$ and $\psi_{6}^{\mathrm{e}}$ equal to zero. In the hcp phase, we have $\psi_{4} \approx 0, \psi_{6} \approx 1$

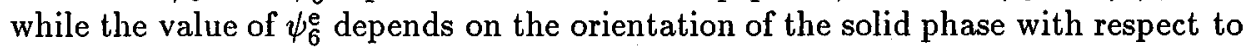
the surface lattice. In general, $\psi_{6} \geq \psi_{6}^{\mathrm{e}}$, and when $\psi_{6}^{\mathrm{e}}$ is considerably lower than $\psi_{6}$ it indicates that a preferred orientation of the adsorbate with respect to the substrate does not coincide with the substrate symmetry axes $[18,19]$. Finally, in the case of uniaxially ordered phase we expect that the bond-orientational order parameters will take some nontrivial values between 0 and 1 .

Besides, we also define the corresponding susceptibilities

$$
\chi_{k}=L_{x} L_{y}\left[\left\langle\psi_{k}^{2}\right\rangle-\left\langle\left|\psi_{k}\right|\right\rangle^{2}\right] / T^{*}, \quad k=4,6
$$


as well as the fourth-order cumulants

$$
U_{k}(L)=1-\frac{\left\langle\psi_{k}^{4}\right\rangle}{3\left\langle\psi_{k}^{2}\right\rangle^{2}}, \quad k=4,6
$$

The simulations have been carried out using the standard Monte Carlo method in the canonical ensemble for three different sizes of the simulation cell: $14 \times 16,21 \times 24$ and $28 \times 32$ (the unit of length is the length of the surface lattice vector $a$ ) and containing different number of particles equal to 224, 504 and 896, respectively, so that the number density was always the same. Standard periodic boundary conditions have been applied. Of course, the system sizes considered here prevent development of long-range correlations and hence we cannot study dynamics of defect pairs. Therefore our analysis must be treated as only preliminary and qualitative. The primary aim of this paper is to demonstrate the usefulness of finite size analysis of higher moments of the bond-orientational order parameters in studies of the systems considered here.

Figure 1 presents the bond-orientational order parameters $\psi_{4}$ and $\psi_{6}$ for the systems of different ${ }^{\circ}$ corrugation of the gas-solid potential and which exhibit the registered $1 \times 1$ structure at low temperatures. No finite size effects are observed

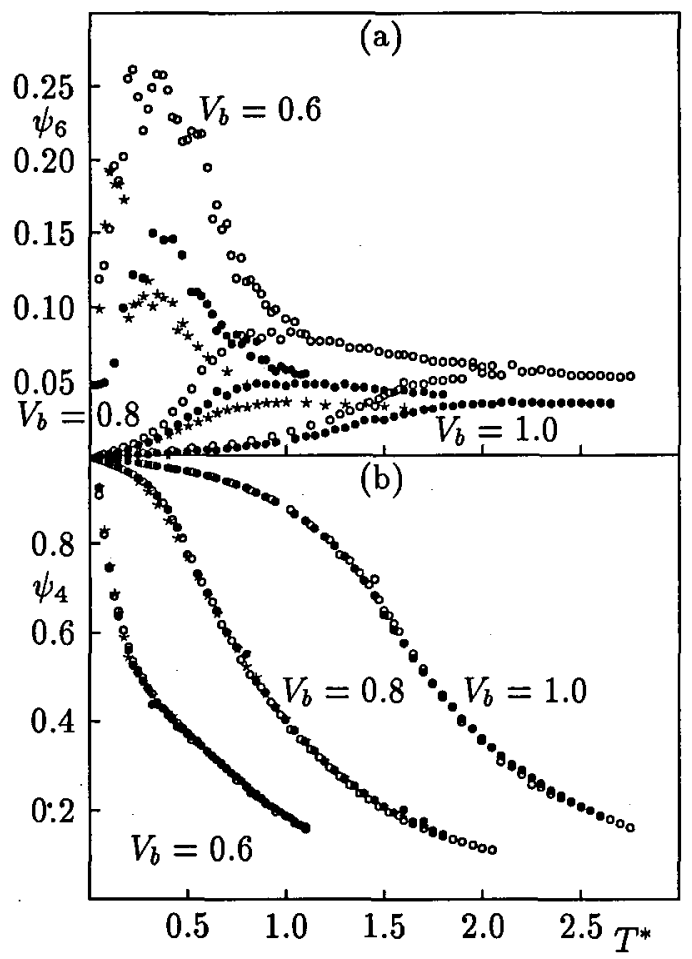

Fig. 1. The bond-orientational order parameters $\psi_{6}$ (part (a)) and $\psi_{4}$ (part (b)) for the systems exhibiting the registered $1 \times 1$ structure at the ground state, obtained for systems of different size ( $\circ N=224, \bullet N=504$ and $\star N=896$ ). 
and the disordering occurs gradually as the temperature grows. Only the behavior of the residual hexagonal order (measured by $\psi_{6}$ ) is affected by the finite size of the simulation cell. The decrease in $\psi_{6}$ for larger systems merely reflects the fact that we are gradually approaching the thermodynamic limit, where $\psi_{6}$ should be close to zero in the both registered and disordered phases. In the case of $V_{b}=0.6$ the system exhibits uniaxial ordering at low temperatures, which gradually disappears as the temperature increases.

In the case of lower corrugation of the gas-solid potential $\left(V_{b}=0.3\right)$ the low temperature solid phase has the hcp structure. Here we find a sharp first-order melting transition located at $T^{*} \approx 0.3$. Although the bond-orientational order parameter $\psi_{6}$ does not exhibit a discontinuity at the transition point (see Fig. 2a), the smooth behavior results from the strong finite size effects. A direct confirmation of the first-order character of melting comes from the behavior of the fourth-order cumulants $U_{4}(L)$ and $U_{6}(L)$. The cumulants for systems of different size should exhibit a common intersection point for any first-order transition $[20,21]$. The results given in Fig. 3 show that both $U_{4}(L)$ and $U_{6}(L)$ possess common intersection points at the reduced temperature $T^{*} \approx 0.3$.

It is noteworthy that the bond-orientational order parameter $\psi_{4}$ considerably increases upon melting (see Fig. 2b). Thus, the liquid phase appears to be partially ordered due to the effects of the surface potential. On the other hand, the bond-

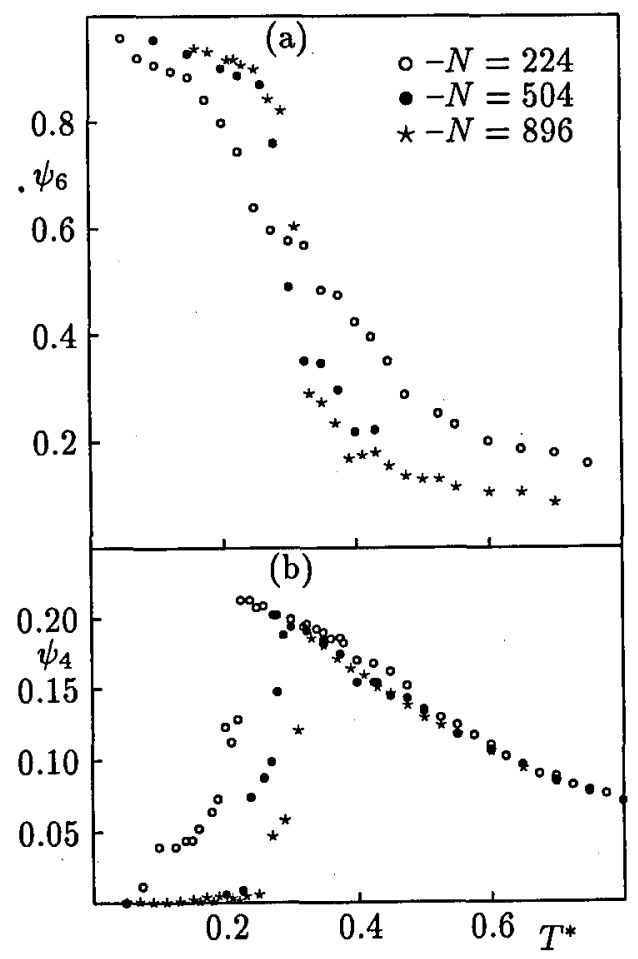

Fig. 2. The bond-orientational order parameters $\psi_{6}$ (part (a)) and $\psi_{4}$ (part (b)) for the systems with $V_{b}=0.3$ and of different size of the simulation cell. 


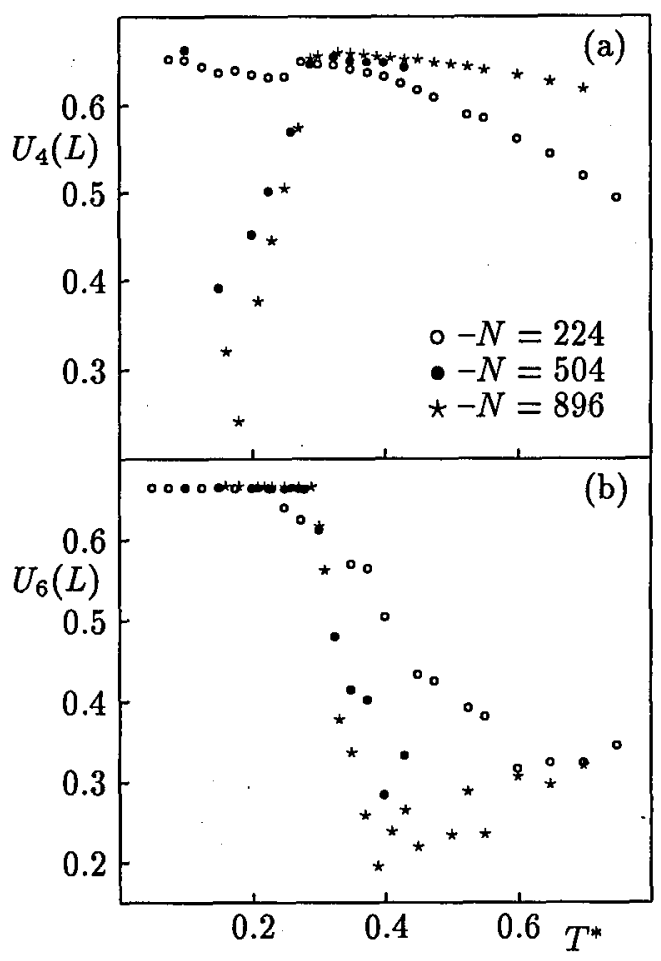

Fig. 3. The fourth-order cumulants $U_{4}(L)$ (part (a)) and $U_{6}(L)$ (part (b)) for the systems with $V_{\mathrm{b}}=0.3$ and of different size of the simulation cell.

orientational order parameter $\psi_{6}^{\mathbf{e}}$ (not presented here) is practically the same as $\psi_{6}$ over the entire temperature range. This shows that the adsorbate layer orientation coincides with one of the symmetry axes of the substrate surface.

For still weaker corrugation of the surface potential $\left(V_{b}=0.1\right)$ the mechanism of melting changes again and appears to be a two-stage process. Figure 4 presents the behavior of the bond-orientational order parameters $\psi_{6}$ and $\psi_{6}^{\mathrm{e}}$ for the largest systems considered here. The results are quite consistent with the predictions of the KTHNY theory $[6,7]$. At $T^{*} \approx 0.4$ we observe a sudden, though small, drop of the bond-orientational parameters. Thus, the system retains a considerable orientational order at temperatures above this transition. Then, the second transition occurs at $T^{*} \approx 0.485$, which is connected with the loss of orientational order in the system. The KTHNY theory predicts that the melting of a two-dimensional film on a weakly corrugated square substrate should be a two-stage process. The first transition, connected with the dissociation of dislocation pairs, transforms the solid phase into hexatic phase, quite similar as in the case of a smooth substrate. The subsequent, disclination-unbinding transition predicted for the smooth substrate, should be replaced by an Ising-like transition. Indeed, from the behavior of the cumulants $U_{6}(L)$ obtained for systems of different size (see Fig. 4) we find that a common intersection point $U^{*}$ occurs at $T^{*} \approx 0.485 \pm 0.005$ and $U_{6}^{*} \approx 0.61$ 


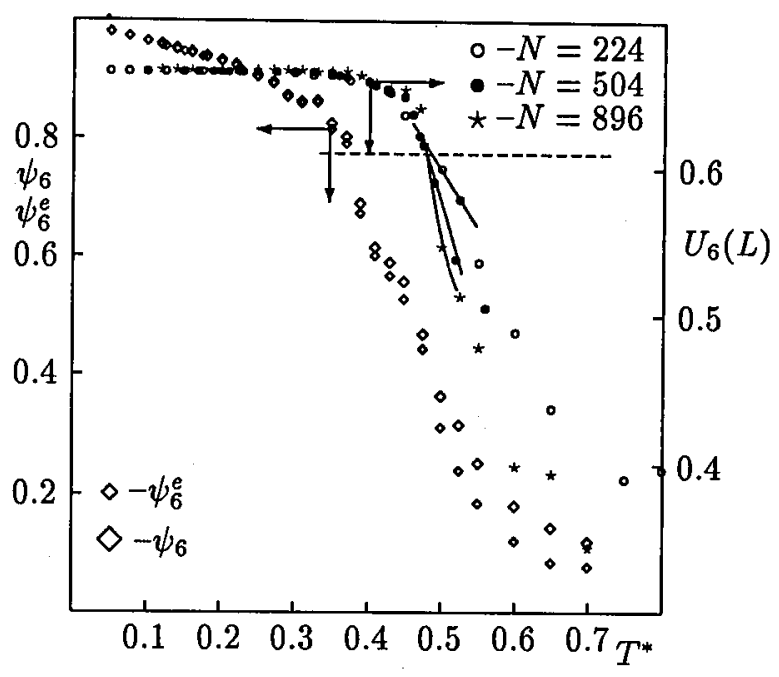

Fig. 4. The bond-orientational order parameters $\psi_{6}$ and $\psi_{6}^{\mathrm{e}}$ for the system with $V_{\mathrm{b}}=0.1$ and the size of the simulation cell $32 \times 28$ containing 894 particles as well as the fourth-order cumulants $U_{6}(L)$ for the same system but for different size of the simulation cell.

has the value corresponding to the universality class of the two-dimensional Ising model $[20,21]$. Of course, we do not claim that the mechanism of melting on weakly corrugated surfaces corresponds to the solid-hexatic-fluid sequence predicted for uniform two-dimensional systems. Our finding, however, is the first clear hint that the mechanism of melting on weakly corrugated square substrate might be the same as predicted by the theory due to Nelson and Halperin [6]. Also, the observed first-order melting on the surface with higher corrugation $\left(V_{\mathrm{b}}=0.3\right)$ is consistent with the prediction of that theory. Although the reported simulation results have been obtained for rather modest system sizes, the presented analysis sufficiently supports our general and qualitative conclusions. To determine the nature of the intervening phase, appearing between the fluid and solid phases, further studies involving calculation of the bond-orientational correlation function and still larger systems are needed.

\section{Acknowledgments}

- A grant for computer time by the ICM (Warsaw University, Poland) is gratefully acknowledged.

\section{References}

[1] K.J. Strandburg, Rev. Mod. Phys. 60, 161 (1988).

[2] W. Janke, Intl. J. Theor. Phys. 29, 1251 (1990).

[3] Y. Saito, Phys. Rev. Lett. 48, 1114 (1982). 
[4] M. Kosterlitz, D.J. Thouless, J. Phys. C 6, 1181 (1973).

[5] B.I. Halperin, D.R. Nelson, Phys. Rev. Lett. 41, 121 (1978).

[6] R.D. Nelson, B.I. Halperin, Phys. Rev. B 19, 2457 (1979).

[7] A.P. Young, Phys. Rev. B 19, 1855 (1979).

[8] J.G. Dash, Films on Solid Surfaces, Academic Press, New York 1975.

[9] P.A. Heiney, P.W. Stephans, R.J. Birgeneau, P.M. Horn, D.E. Moncton, Phys. Rev. $B$ 28, 6416 (1983).

[10] Y. Larher, in: Surface Properties of Layered Structures, Ed. G. Benedek, Kluwer Academic Publishers, Netherlands 1992, p. 261.

[11] F.F. Abraham, Phys. Rep. 80, 339 (1981).

[12] Bond-Orientational Order in Condensed Matter Systems, Ed. K.J. Strandburg, Springer, Berlin 1992.

[13] H. Weber, D. Marx, K. Binder, Phys. Rev. B 51, 14636 (1995).

[14] A. Patrykiejew, S. Sokolowski, T. Zientarski, K. Binder, J. Chem. Phys. 102, 8221 (1995).

[15] W.A. Steele, Surf. Sci. 36, 317 (1973).

[16] H.-Y. Kim, W.A. Steele, Phys. Rev. B 45, 6226 (1992).

[17] L.W. Bruch, J.A. Venables, Surf. Sci. 148, 167 (1984).

[18] A.D. Novaco, J.P. McTague, Phys. Rev. Lett. 38, 1286 (1977).

[19] J.P. McTague, A.D. Novaco, Phys. Rev. B 19, 5299 (1979).

[20] K. Binder, Z. Phys. B 43, 119 (1981).

[21] K. Vollmayr, J.D. Reger, M. Scheucher, K. Binder, Z. Phys. B 91, 113 (1993). 